Revue des patrimoines

40 | 2019

Lits historiques. Première anthologie des lits européens du XVe au XIXe siècle

\title{
Le lit en France à la fin du Moyen Âge. Symbolique sociale dans les sources notariales
}

The bed in France in the late Middle Ages. The social Symbolic in the notarial sources

\section{Donatien Guégan}

\section{OpenEdition}

Journals

Édition électronique

URL : http://journals.openedition.org/insitu/22584

DOI : $10.4000 /$ insitu.22584

ISSN : 1630-7305

\section{Éditeur}

Ministère de la Culture

\section{Référence électronique}

Donatien Guégan, «Le lit en France à la fin du Moyen Âge. Symbolique sociale dans les sources notariales », In Situ [En ligne], 40 | 2019, mis en ligne le 15 septembre 2019, consulté le 04 octobre 2019. URL : http://journals.openedition.org/insitu/22584; DOI : 10.4000/insitu.22584

Ce document a été généré automatiquement le 4 octobre 2019.

\section{(i) $\odot$

In Situ Revues des patrimoines est mis à disposition selon les termes de la licence Creative Commons Attribution - Pas d'Utilisation Commerciale - Pas de Modification 4.0 International. 


\section{Le lit en France à la fin du Moyen Âge. Symbolique sociale dans les sources notariales}

The bed in France in the late Middle Ages. The social Symbolic in the notarial sources

Donatien Guégan

1 Dans l'historiographie française, le lit a souvent été considéré comme un objet physique, de représentation et d'usage. Nombre de chercheurs et chercheuses ont fait apparaître la variété des usages du lit médiéval. Les travaux sur l'iconographie domestique menés par Françoise Piponnier ${ }^{1}$, Perrine $\mathrm{Mane}^{2}$ et Danièle AlexandreBidon $^{3}$ ont développé la compréhension des pratiques autour du lit, de jour comme de nuit. Véritable objet diurne à part entière dans l'habitat, le lit était ainsi tantôt banc, tantôt tapis ; symbole du pouvoir seigneurial, lit de parade et lit de justice. La nuit était aussi l'occasion pour le lit d'être au centre de l'attention domestique; réceptacle de l'intimité, qu'elle soit conjugale ou extra-conjugale, du malade, du mourant. Mais le lit peut s'avérer aussi le témoin de bien d'autres pratiques de l'ordre de la symbolique sociale que les textes, en particulier les actes notariés, permettent de mettre en exergue. Ces éléments éclairant l'histoire matérielle, jusque-là quasiment inexplorés, méritent qu'une première analyse en esquisse l'ampleur et les possibilités pour la recherche historique.

2 Objet de tous, le lit est, avec le coffre et le buffot, un meuble constituant le socle fondamental de l'habitat médiéval, comme le prouve cet inventaire dijonnais du modeste Robert d'Estevaulx qui dispose de bien peu de mobilier, mais tout de même de l'essentiel :

Une archote [petit coffre] de fol (autre graphie pour «foul »: hêtre), 1 autre viez arche de chaigne, deux selles a seoir sur, 1 sofflot, 1 buffot, 1 soillot [seau] ferre, 1 corde poiseure, II viez poinsons, 1 XII $^{\text {ne }}$ d'escuelle de bois, 1 mortier de pierre, 1 pestot [pilon], une ansote [ustensile à anse], 1 cromasle [crémaillère], 1 meschant 
treppier de fer a deux piez, 1 lit de queneton [duvet] garni de coussin de queneton,

deux linceulx et une meschant coustrepointe... ${ }^{4}$

Dans la seule pièce qui constitue le logement du défunt peuvent être retrouvés tous les objets usuels: un lit, deux coffres, quelques ustensiles, deux assises, et ce buffot à surface plane permettant de poser des objets, de manger, travailler (fig. 1).

Figure 1

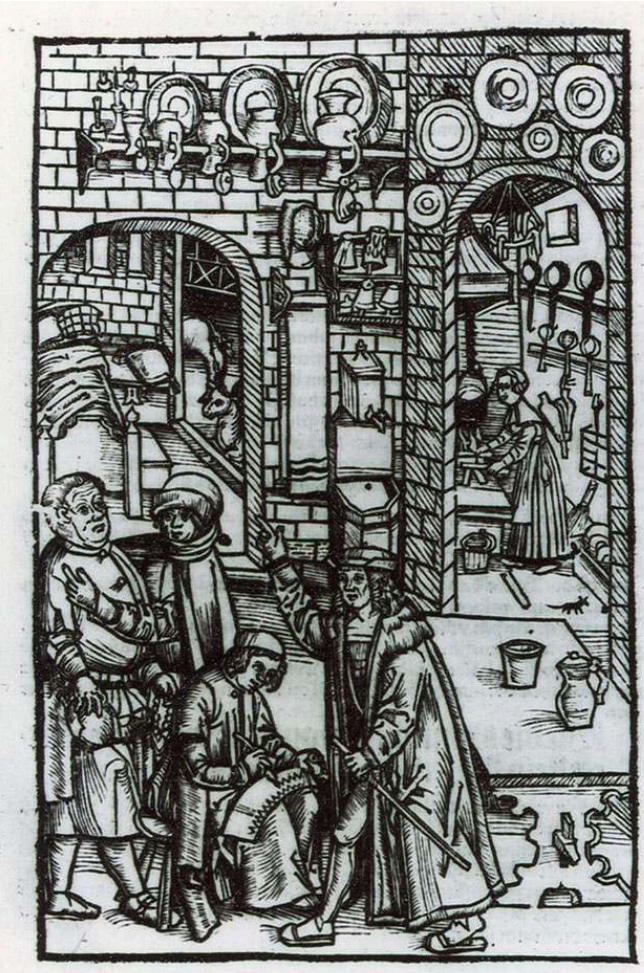

Code pénal de Bamberg, gravure sur bois, 1510. Paris, BnF, Rés. F 667 fol $11 \mathrm{~V}$.

Repro. Donatien Guégan, 2018. (c) BnF.

Mais au-delà de cet aspect commun, qui a déjà été traité dans des travaux marquants, le lit est un meuble à la symbolique très forte. Il serait vain de prétendre à l'exhaustivité, cependant, nous nous appuyons ici sur différentes recherches effectuées dans le cadre d'un mémoire de master centré sur les usages et les prix du mobilier en France à la fin du Moyen Âge ${ }^{5}$. Si celui-ci s'intéressait principalement aux meubles de rangement (aulmaires - armoires -, coffres, buffots et matherots - buffets) et ceux liés à l'espace culinaire, ces recherches dans les archives d'une dizaine de départements ont permis de découvrir des actes notariés où le lit est présent, et ainsi d'y distinguer des usages sociétaux très intéressants qui méritent d'être développés ici.

\section{Le lit, objet de legs personnel}

5 Tout d'abord, s'il est un objet de legs, c'est bien le lit; cette qualité apporte d'ailleurs parfois des informations sur le lit en tant que meuble dans son usage. En 1420 par exemple, Jean Julien lègue à sa femme un «leda arche» [lit coffre $]^{6}$. Il s'agit certainement d'un lit fermé, constitué d'une sorte de grand coffre en bois clos par des portes à battant, voire coulissantes, tels qu'il en a existé fréquemment dans les 
campagnes aux $\mathrm{xIX}^{\mathrm{e}}$ et $\mathrm{Xx}^{\mathrm{e}}$ siècles. Cette forme de lit tout à fait inédite pour le Moyen Âge, a été signalée une fois, par Philippe Maurice, en Gévaudan ${ }^{7}$. Avec d'autres études, peut-être que de nouveaux exemples de ce type de couche seront rapportés, et qu'il sera possible d'analyser à quelle échelle il a été utilisé, et à quand cette forme remonte.

Plus intéressant encore, lorsque le lit est mentionné dans les testaments, il s'agit en général de l'unique meuble, ou ensemble de meubles (dans le cas de dons de textiles liés au lit, notamment des courtepointes) qui est légué. Il est en effet extrêmement rare de voir des legs de vaisselle, de coffre ou d'autre mobilier, quelle que soit la classe sociale. Dans les testaments de Paris que l'on peut exploiter, que ce soit ceux conservés aux Archives nationales ou ceux qui ont été édités par Alexandre Tuetey ${ }^{8}$, le fait de léguer un lit répond à deux démarches distinctes mais parallèles. Premièrement, le legs aux proches : «Item, elle laissa à sa mere deux liz et deux coutepointe qui estoient chiez Estienne Coustet, clerc du tresorier de monsieur le duc d'Orleans ${ }^{9}$. »

Ou cet autre exemple :

Item, à Jehanneton, ma chamberiere, qui nous a servis par longtemps, pour le bien et avancement de son mariage, je lui laisse dix fr., ung lit, une couverture moyenne, deux paires de draps de deux lez de chanvre, deux cuevrechiefz, ung orillier et lui laisse dix aulnes de drap pour la vestir à ses nopces du pris d'ung escu l'aune, et se ainsi est que lad. Jehanneton face aucune chose qui ne soit pas à point ou qu'elle ne se mariast au gré de ses amis et parens, je vueil le don estre nul et aussi que les executeurs ne distribuent à ame de ce que est dit au dessus jusques que elle ait trouvé partie par nom de mariage ${ }^{10}$.

8 Le premier legs, d'une fille à sa mère et datant du 21 juillet 1414, n'a rien d'exceptionnel en soi, bien qu'il atteste que les enfants pouvaient souhaiter laisser à leurs parents des biens, puisque le cas du décès de l'enfant émancipé avant les parents n'est pas courant dans les sources médiévales. Dans le cas d'une veuve, le premier bénéficiaire semble être le parent, à qui Marie lègue son lit. Le deuxième cas, daté du 26 octobre 1420, est également intéressant, puisqu'il s'agit d'un legs sous forme de trousseau pour un mariage futur. Il est tout à fait important pour la compréhension des liens qui unissent maître et serviteur. Ici, la maîtresse exige une certaine attitude de la part de sa servante même si elle n'est plus à son service, en cherchant à travers son testament à conserver une certaine autorité morale sur sa chambrière; si celle-ci ne se comporte pas selon les préceptes chrétiens, et ne se marie pas « au gré de ses amis et parens ", le don sera annulé. Ainsi, le lit, par le biais du don, se fait l'expression d'une morale chrétienne à laquelle se conforme le mourant devant le trépas, en plus des traditionnelles aumônes pour son âme. À travers le souci de moralité qu'elle manifeste à l'égard de sa servante, Simonette La Maugère veut perpétuer le souvenir de sa propre piété (fig. 2). 
Figure 2

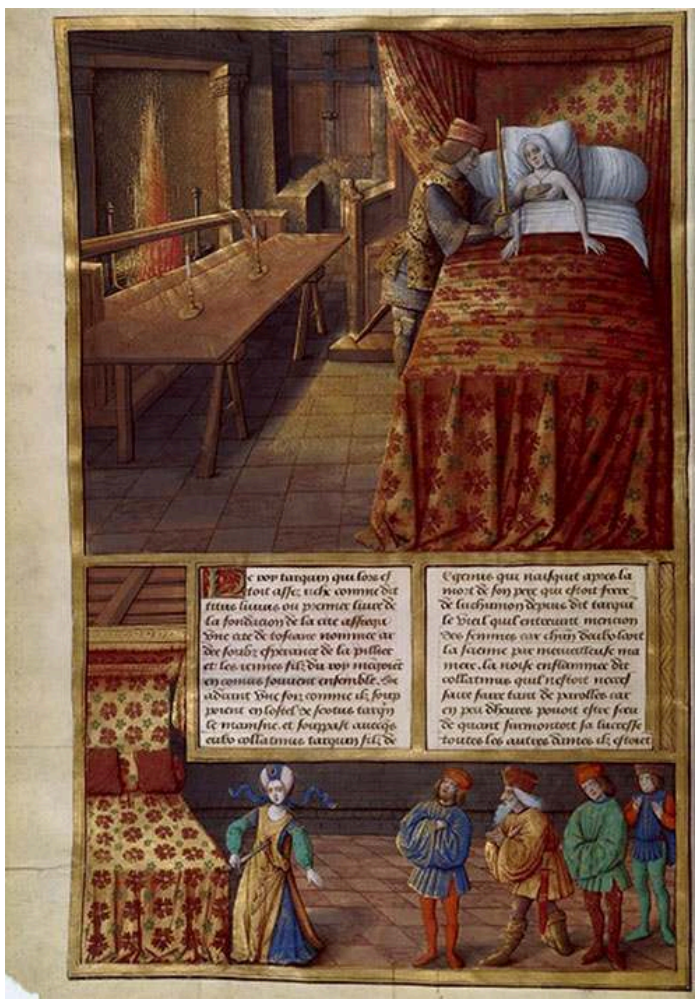

«Viol et suicide de Lucrèce », dans Benvenuto Rambaldi, Romuleon (trad. Sébastien Mamerot). Paris, $\mathrm{BnF}$, ms Français 365 , fol $^{\circ} 27 \mathrm{v}$.

(C) Base Mandragore, BnF.

Les dons d'un maître à ses serviteurs sont assez fréquents dans les testaments parisiens et ils donnent une idée de la richesse des relations qui se nouaient entre maîtres et serviteurs.

Item, le dit testateur laissa à Perrin d'Auxy, son serviteur, le lit où icelui testateur gisoit, c'est assavoir, couste, coissin, un gros oreillier, deux paire de draps, dont l'une sera neufve, et l'autre des draps communs de son hostel, et une coutepainte, sans ciel, sans dossier, et sans trois custodes noires qui sont sur le dit lit ${ }^{11}$.

Item, je laisse oultre au dit Perrin Josse $\mathrm{III}^{\mathrm{c}}$ escus d'or, un de mes liz estoffé de deux draps et couverture, tel qu'il vouldra prandre après le meilleur, six tasses blanches, VI cuilliers d'argent, six hanaps de madre [bois] après les deux meilleurs, une douzaine d'escuelles d'estain, quatre plas moyens, deux salieres d'estain et deux chandeliers.

Item, à Girardin Quinote qui me sert, ou cas qu'il sera avecques moy quant je morray, II livres tournois, un petit lit estoffé, et ne demande riens pour cause de salaire, car aussi l'ay-je assez satisfait, vestu et norry, et ou cas qu'il ne seroit point avecques moy, X livres tournois seulement. Item, à chascun de mes autres serviteurs $\mathrm{X}$ livres tournois ${ }^{12}$.

Ce dernier cas est particulièrement pertinent pour cette étude. Jean de Neuilly-SaintFront, chanoine de Notre-Dame de Paris et archidiacre de Soissons, personnage important, a un fils adoptif, Jean Damade. Il lui laisse tous ses biens immobiliers puis fait des dons à ses six autres serviteurs. Mais ce sont deux d'entre eux en particulier qui semblent recueillir ses faveurs. Le premier, Perrin Josse, reçoit un don de trois cents écus, une somme relativement importante, mais également son deuxième meilleur lit (on ne sait malheureusement pas ce qu'il advient du premier, apparemment 
encore plus symbolique; il est vraisemblablement légué à Jean Damade), et de la vaisselle. Le prix d'un lit de qualité, s'il peut être important ${ }^{13}$, n'est jamais excessif ; une somme de trois cents écus d'or équivaut largement à trente fois ce que Jean de NeuillySaint-Front donne à Josse en mobilier, aussi la motivation du donateur n'est pas d'enrichir son légataire. Est-ce pour marquer un lien particulier avec Perrin Josse, que la monnaie, reproductible et froide, ne suffirait pas à symboliser ? C'est en tout cas une hypothèse, que ce don permet de formuler (fig. 3).

Figure 3

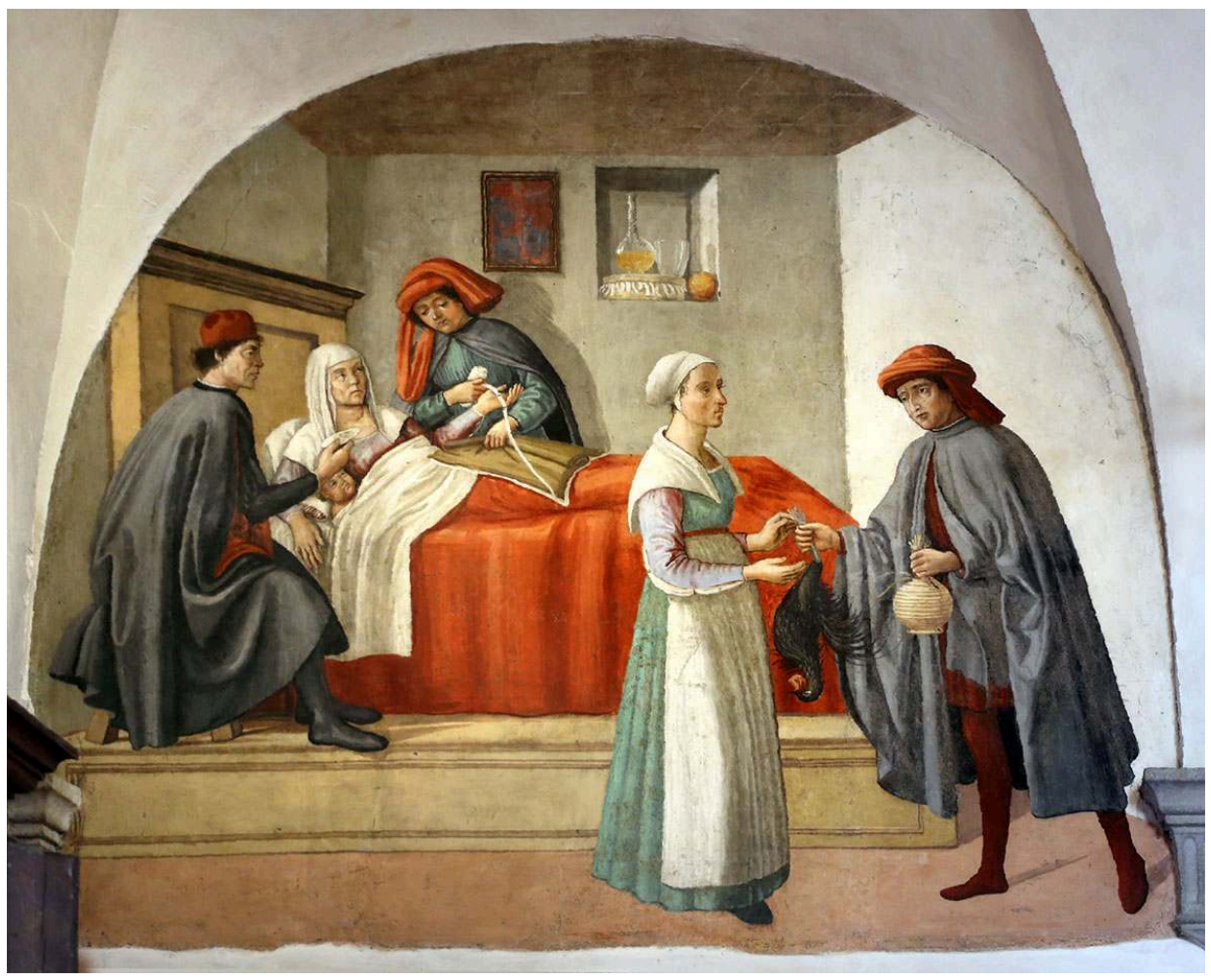

Femme malade alitée soignée par des médecins, fresque. Oratorio dei Buonomini di San Martino, Florence, Italie, vers 1478.

(C) SAILKO, 2018.

11 Le second legs cité est tout aussi intéressant pour la précision que le testateur prend soin d'apporter. Il spécifie clairement «ou cas qu'il sera avecques moy quant je morray » et " car aussi l'ay-je assez satisfait, vestu et norry ». Il semble que le testateur ait quelque réserve vis-à-vis de ce serviteur, qu'il apprécie assez pour le traiter mieux que les autres, et envers qui il rappelle s'être montré très généreux ; quelque méfait à lui reprocher? Peut-être doute-t-il de sa loyauté totale, et ne lui accordera-t-il son entière confiance que s'il reste avec lui jusqu'à sa mort; alors il sera récompensé comme il le prévoit. Est-ce parce que ce serviteur a déjà quitté son service une fois ? Quoi qu'il en soit, la sentence est sans appel : s'il reste, il recevra deux cents livres et un lit garni mais s'il part, il sera traité à l'égal des autres et ne recevra "que » dix livres tournois. Cette différence de traitement reflète probablement des liens, des rapports différents, comme l'atteste le legs initial.

Donner un lit semble un geste infiniment symbolique. On lègue les biens immeubles et ce qu'ils contiennent aux héritiers principaux, un lit aux principaux serviteurs, comme 
une marque de respect, et de l'argent en espèces aux autres, don qui n'a pas forcément une valeur intrinsèque plus faible que le lit, mais qui est moins personnel et beaucoup moins intime et signifiant.

\section{Le lit, premier meuble du don pieux}

13 À propos de don personnel, considérons à présent le legs pieux de lit. En effet, quel don plus sacré que le lit " où gisoit le défunt », dernier vestige terrestre du fidèle avant son rappel à Dieu. Là encore, les informations que recèlent les testaments parisiens nous sont d'un grand secours :

Item, lad. testaterresse laissa aux povres des hospitaulx de Nostre-Dame du Puy et de Saint-Laurens deux lis garnis de couste, de coissins, de orriliers, deux paires de draps chascun lit et une couverture armoré de ses armes, c'est assavoir un lit à chascun desd. hostieaulx garni comme dit est ${ }^{14}$.

Item, à l'Ostel-Dieu de Paris le plus grant lit et le meilleur qu'elle aura au jour de son trespassement, garni de deux paires de draps de lin les plus beaux et les meilleurs qu'elle ait, deux oreilliers et deux quouvrechiés et une chambre, laquelle sera tendue sur le lit où elle trespassera ${ }^{15}$.

Item, je laisse à l'Ostel-Dieu de Paris, tant à l'office de maistre comme à l'office de la prieure, par moitié vint fr. avec la chambre et le lit entier auquel je trespasseeroy ${ }^{16}$.

Dans ces trois exemples, les donateurs sont très aisés, et donnent des centaines, voire des milliers de livres à différentes œuvres de charité pour le salut de leur âme. Mais le seul meuble que le riche testateur lègue à une institution charitable, c'est son lit. Dans le premier cas, ce sont apparemment deux lits à l'appartenance indéterminée qui sont donnés à deux hôpitaux, mais dans les deux autres, les testatrices offrent leur propre lit à l'Hôtel-Dieu de Paris.

Dans une organisation testamentaire aussi codifiée que l'était celle des classes aisées au Moyen Âge ${ }^{17}$, il est difficile de croire à une coïncidence. Marie du Bois, la première des deux testatrices données en exemple, fait des legs pieux à de nombreux hôpitaux, maladreries et couvents parisiens, pour un montant total de trois cent vingt-cinq livres et quatorze sous parisis. L'Hôtel-Dieu, lui, ne reçoit aucun don en numéraire, seulement le lit de la testatrice. C'est, d'après cette étude, dans une logique hautement symbolique que les deux femmes lèguent ainsi un bien très personnel, et la seconde précise même "le lit entier auquel je trepasseeroy ». Ce n'est pas un lit dont elle n'a pas l'usage ou qu'elle ferait acheter pour le léguer, mais le sien, dans lequel son corps quitte le royaume terrestre pour le royaume céleste (fig. 4). 
Figure 4

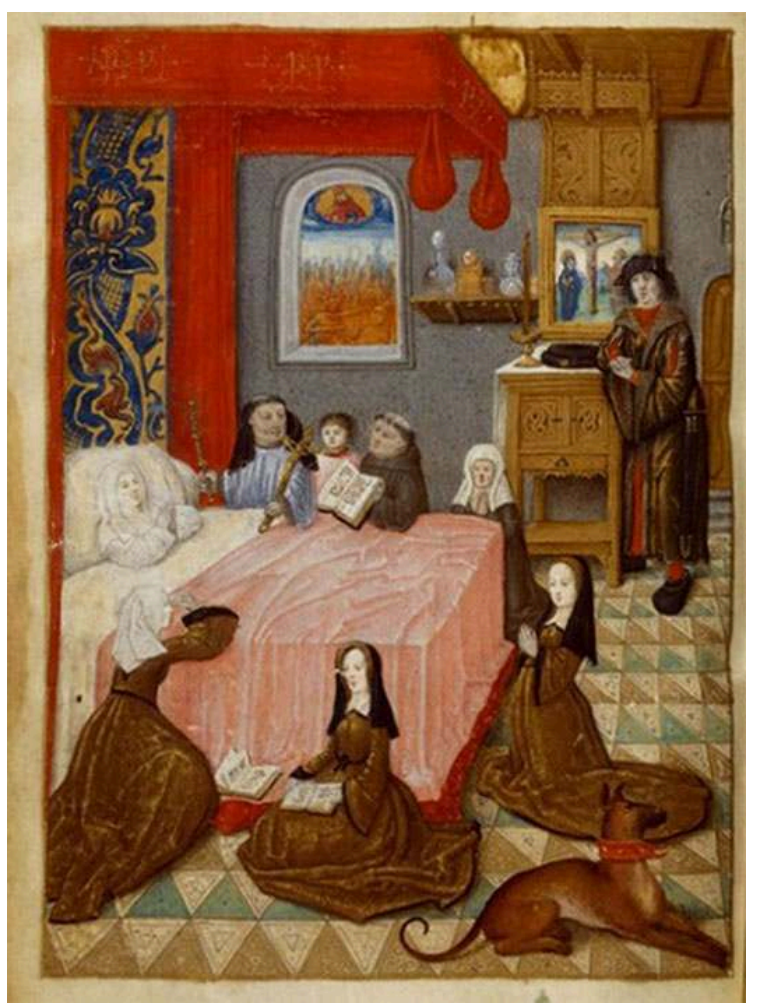

Bernard de Clairvaux, Livre d'heures, en latin et en français, à l'usage de Cambray. Paris, Arsenal, ms 1185, fol $^{\circ} 373 v$. Gallica.bnf.fr.

(C) BnF.

Il serait à ce sujet extrêmement enrichissant de savoir si les hospices ainsi honorés se prévalaient d'un tel don et de quelle manière. Dans la même idée, il serait instructif de savoir si ces lits et leur riche garniture étaient revendus afin d'acquérir du mobilier plus ordinaire mais plus utile, ou s'ils étaient conservés sans être modifiés et employés tels quels. Une étude poussée des documents concernant les établissements légataires ainsi que l'analyse et la lecture des travaux de chercheurs comme Christine Jehanno semblent tout indiquées dans cette optique ${ }^{18}$.

Si les libéralités testamentaires fournissent des lits aux proches et aux établissements de charité, le lit est également, assez logiquement, un présent de choix lors des unions.

\section{Le lit, symbole de l'alliance matrimoniale}

En effet, cette valeur symbolique du lit, quel moment plus approprié dans une vie où l'honorer qu'un mariage? L'édition pour l'année 1437 des actes de l'étude du notaire orléanais Pierre Christofle réalisée par Kouky Fianu (mise en ligne par l'École nationale des chartes depuis 2015) est une avancée considérable pour la recherche dans les sources textuelles médiévales ${ }^{19}$. C'est un exemple qu'il convient de suivre, et les riches fonds notariés conservés aux archives départementales du Loiret nous ont semblé un corpus séduisant pour amorcer une édition de textes sur l'histoire matérielle. Les extraits ci-dessous montrent bien le rôle prépondérant du lit tel qu'il apparaît dans les contrats de mariage en Orléanais au $\mathrm{Xv}^{\mathrm{e}}$ siècle : 
Et par ledit mariage faisant, ledit Pierre Morant a promis donner et bailler audit Jehan Gaultier, tant pour toute telle partie, porcion et eschouatte de biens meubles qui povoient compecter et appartenir a ladicte Jehanne par le trespas dudit feu Robin Toubert son pere, comme pour tous louaiges et services que ladicte Jehanne a peu avoir faiz et destinz depuis le trespas de sondit feu pere avec le demourant et sadicte mere et aultrement de tout le temps passé jusques a huy, la somme de trente livres tournois monnoye courant a present, avec ung lit g[arnis] de coeste, coessin, une couvertur, quatre draps de lit [illisible] de deux lez, quatre quevrechiefs, quatre nappes, quatre [illisible] touailles tout bon linge et convenable et ung coffre tout neuf, une cotte hardie de drap noir fourree bien et honnestement selon son estat et une cotte simble vermeille pour le jour de sa veneicon [mariage] et ung traversain ${ }^{20}$ de vin vermail pour faire les nopces ${ }^{21}$.

C'est assavoir que ledit Jehan Blanchart promect prandre ladicte Marion. Et Mahiet Lerant, frere de mere de ladicte Marion, a promis lui fere avoir a femme se Dieu et Saincte eglise sy accordent, et plus par ledit traicte de mariage faisant ledit Mahier, ou nom [de] sadicte seur, sy a promis donner et bailler audit Blanchart, a cause de ladicte Marion, la somme de vint livres tournois monnoie courant a present, avec une cote simple vermeille, une cote de drap non fourree de drap communs, ung lit garny de coeste, coessin, deux couvertures l'une vermeille et l'autre percee, six draps de lit de chanvre, six nappes, six touailles, six queuvrechefs une huche fermant de clef, six escuelles, deux plaz et deux pintes, une d'estain, a la volonte dudit Jehan Blanchart ${ }^{22}$.

Et par ledit traictié de mariage, et pour icellui estre fait et consommé, ledit Huguenin approuva donner et bailler audit Olivier, a cause de sadicte fille, la somme de douze livres tournois, monnoie courant a present, lesquielx XII livres tournois ledit Huguenin paiera et sera tenu paier audit Olivier, c'est assavoir promptement X livres tournois et le seurplus et demourant montant a II frans a la feste de Saint Jehan Baptiste prouchainement venant. Sera tenu avec ce bailler aux nopces dudit Olivier une cote simple et une cote hardie, ung lit garni de coeste, coessin, couverture, IIII draps, II queuvre chefs, ung orillier, II nappes et II touailles ${ }^{23}$. entre deux parties, les actes notariés du Loiret offrent un autre exemple d'engagement social où le lit prend une place symbolique de choix, le contrat d'apprentissage : « Et lui 
paieront franchemment et quictement la somme de [dix : biffé] quatorze livres tournois marc d'argent à VII livres tournois et ung lit de deux lez garny de coeste, coessin, couverture et deux draps bons et convenables ${ }^{24}$. »

En acceptant de prendre un jeune cousin âgé de cinq ans pour le former au métier de la vigne, un viticulteur promet de le payer une certaine somme sur douze ans, mais aussi de lui fournir un lit garni. Considérant le contrat d'apprentissage comme un lien, en particulier lorsqu'il s'établit, comme fréquemment, à l'intérieur de la famille, l'engagement à fournir un lit lors de l'entrée en apprentissage du jeune, lit qu'il pourra conserver au terme de cette période, lorsqu'il aura dix-sept ans, apparaît réellement comme le dernier don du maitre afin de lancer le novice dans la vie. Le lit garni se manifeste ici comme une métaphore du foyer, que le formateur offre à son apprenti au moment où celui-ci quittera son aile protectrice.

Dans une réflexion plus pratique que symbolique, le lit est ici le meuble primordial, qui peut suffire un temps à garnir un foyer modeste, comme évoqué en début d'article, ou être, dans le cas d'un futur journalier, le moyen de garantir une paillasse de qualité s'il venait à être logé chez son employeur.

Un autre exemple particulièrement intriguant est celui de deux actes inédits de l'étude d'un notaire de Villepreux. Se retrouvent ainsi chez le notaire un boulanger et un autre homme pour deux actes, à deux mois d'intervalle. Le premier consiste en un bail de vaisselle d'une durée d'un an: le boulanger loue six écuelles et un saulcier (récipient pour sauces) ${ }^{25}$ à un certain Jaquet, qui se dit tavernier, puis deux mois plus tard, en qualité de maître hôtelier, le même Jaquet loue au même boulanger pour un an un lit garni d'un traversin, de coussins et de couvertures ${ }^{26}$. D'une part, il est intéressant de constater que le preneur change d'emploi selon ce qu'il loue, on peut émettre l'hypothèse qu'il spécifie une activité en rapport avec l'objet loué (tavernier quand il emprunte de la vaisselle, hôtelier quand il loue un lit garni) ou tout simplement qu'il a fait évoluer ou souhaite faire évoluer son activité. Mais le plus étonnant est que l'on baille du mobilier, en l'occurrence notre objet d'étude, un lit. Cet exemple nous paraît tout à fait inédit dans l'histoire matérielle, et demanderait une analyse plus fine et des recherches dans d'autres fonds. Pour quelle raison ce tavernier-hôtelier a-t-il loué pour un an ces objets? D'autant que dans le second cas, cela lui coûte vingt sous parisis, somme non dérisoire. Avec une telle somme, il aurait sans doute pu se procurer un lit neuf; mais peut-être est-ce là le signe d'un manque de liquidités, pallié par une solution à moyen terme, où le preneur ne paye qu'à la fin du bail, soit au bout d'un an ? Serait-ce là le règlement détourné d'une dette contractée auprès du boulanger? Une recherche systématique sur ces deux personnages dans ce fonds nous permettrait peutêtre d'avoir une idée plus précise de leur activité, et pourquoi pas, de ce qui justifie ce contrat. Toujours est-il que, les actes étant passés devant notaire, ils impliquent un lien sociable, moral et légal qui méritait, au moins du point de vue du bailleur, la rédaction d'un contrat authentique malgré les sommes mesurées engagées, car comme l'explique Thierry Dutour, «les relations économiques sont des relations sociales ${ }^{27}$ impliquant réseau et confiance ${ }^{28}$.

\section{Conclusion}

Cette recherche nous a permis de caractériser et de distinguer divers aspects symboliques du lit dans la société de la fin du Moyen Âge en France. Les différentes 
sources exploitées reconnaissent la nature prééminente du lit dans un contexte de liens patrimoniaux et spirituels, dans la sphère privée comme publique.

Les aspects symboliques d'un don couché sur papier il y a plusieurs siècles reposent sur des interprétations, qui tentent malgré tout d'être les plus objectives possible. Dans le cadre de la recherche en histoire matérielle médiévale, le lit commun peut parfois sembler un élément subsidiaire vis-à-vis du lit d'apparat extraordinaire (lit royal, lit de justice). Les faits, accumulés dans les actes, ont semblé toutefois former petit à petit un ensemble cohérent qu'il nous aurait paru dommage de laisser de côté, quelle que soit la difficulté d'analyser l'arrière-plan d'un acte plusieurs siècles après sa rédaction. Notre objectif n'était pas ici tant de savoir si ces dons symboliques ont été pensés, calculés et ordonnés, mais bien d'essayer de comprendre pourquoi ils se sont manifestés simultanément, à différents niveaux de l'échelle sociale, dans de nombreux aspects communs de la vie quotidienne, sous une forme semblable.

Sans nul doute, une meilleure connaissance de ce que représente l'objet lit à travers les sources textuelles permettra une compréhension beaucoup plus fine de l'organisation sociale médiévale.

\section{NOTES}

1. - PIPONNIER, Françoise. «La diffusion des tentures à la fin du Moyen Âge. L'exemple de la Bourgogne ». Dans Ead. (dir.). Tentures médiévales dans le monde occidental et arabo-islamique. Actes de la journée d'étude de Lyon, 16 mars 1994. Mélanges de l'École française de Rome. Moyen Âge, 111/1, 1999, p. 419-442.

2. - MANE, Perrine. "Le lit et ses tentures d'après l'iconographie du XIII ${ }^{\mathrm{e}}$ au Xve $s$. ». Dans ibid., p. 393-418.

3. - ALEXANDRE-BIDON, Danièle, PIPONNIER, Françoise. "Mobilier civil et intérieurs domestiques». Dans PRIGENT, Christiane (dir). Art et société en France au XVesiècle. Paris: Maisonneuve et Larose, 1999, p. 643-656 ; ALEXANDRE-BIDON, Danièle, PIPONNIER, Françoise, POISSON, Jean-Michel (dir.). Cadre de vie et manières d'habiter ( $\mathrm{XII}^{e}-\mathrm{XVI}^{e}$ s.). Caen : Publications du CRHAM, 2006.

4. - FERRAND, Guilhem. Les Inventaires après décès de la ville de Dijon à la fin du Moyen Âge. Toulouse : Presses universitaires du Midi, coll. "Méridiennes", 2018, Inventaire 76 (11 mai 1405).Transcription : «Un petit coffre en hêtre, un autre vieux coffre de chêne, deux sièges sans dossier pour s'asseoir, un soufflet, un buffet, un seau en fer, une corde à puiser l'eau, deux vieilles futailles, une douzaine d'écuelles en bois, un mortier en pierre, un pilon, un petit ustensile à anse, une crémaillère, un trépied en fer à deux pieds de mauvaise qualité, un lit en duvet garni de deux coussins en duvet, deux draps de lit et une couverture de mauvaise qualité. »

5. - GUÉGAN, Donatien. Plustot au buffet qu'à la broche. Utilisation et prix du mobilier en France à la fin du Moyen Âge. Master d'histoire sous la direction de Thierry Dutour. Paris : Sorbonne Université, 2018.

6. - AD Lozère, G 1378/61, Étude Julien.

7. - MAURICE, Phillipe. «La maison et son ameublement en Gévaudan à la fin du Moyen Âge ». Journal des savants, 1998, $\mathrm{n}^{\circ}$ 2, p. 135. 
8. - Testaments enregistrés au Parlement de Paris sous le règne de Charles VI. Éd. Alexandre Tuetey. Paris : Imprimerie nationale, 1880.

9. - Registre S. BnF, Moreau 1161, fol. 772v-778v. Marie, veuve de Pierre Le Cerf, 1414, 21 juillet.

10. - Testaments enregistrés au Parlement de Paris... Éd. cit., p. 382-385 : Simonette La Maugère, 1420, 26 octobre.

11. - Registre R, Arch. nat., $X^{1 A} 9807$, fol. 203. Thomas l'Écorché, licencié ès lois, avocat au Châtelet de Paris, 1406, 13 septembre.

12. - Registre R, Arch. nat., $\mathrm{X}^{1 \mathrm{~A}}$ 9807, fol. 78, Jehan de Neuilly Saint-Front., 9 octobre 1402.

13. - Une étude globale - même régionale - du prix du mobilier serait à n'en pas douter d'un grand apport aux réflexions sur l'aménagement intérieur de l'habitat médiéval, de même que pour toute recherche où du mobilier serait engagé. À Dijon, un lit garni de couette et coussins d'un maître maçon a été prisé 5 francs le 15 mars 1398 (n. st.) (FERRAND, Guilhem. Les Inventaires... Op. cit., Inventaire 32), mais seuls les lits princiers ou royaux peuvent dépasser ces sommes.

14. - Testaments enregistrés au Parlement de Paris sous le règne de Charles VI. Éd. cit., p. 252-264 : Alix de Cournon. 1410 [n. st.], 22 janvier 1410 [n. st.], $1^{\mathrm{er}}$ février.

15. - [Registre R, Arch. nat., $X^{1 A} 9807$, fol. 446v-448, manquants aujourd'hui], Série $T, B n F$, Moreau, 1162, fol. 200-205v. (Mention de collation, fol. 205V, Marie du Bois, 1416, 30 novembre).

16. - Testaments enregistrés au Parlement de Paris sous le règne de Charles VI. Éd. cit., p. 337-346: Marguerite de Bruyères, 1416, 30 juin.

17. - LORCIN, Marie-Thérèse. «La pratique testamentaire en Lyonnais et Forez à la fin du Moyen Âge ». Dans MALAMUT, Élisabeth (dir.). Dynamiques sociales au Moyen Âge, en Occident et en Orient. Aix-en-Provence : Publications de l'université de Provence, 2010, p. 33-41; CHAIGNE, Marion. "Pour le remède et salut de mon âme... ». Édition et commentaire de trente et un testaments de femmes enregistrés au Parlement de Paris (1394-1420). Thèse de l'École nationale des chartes. Paris : 2006 ; ROUSSEL, Stéphanie. "En pur et vray don ». Donateurs et donations pieuses aux Célestins de Paris à la fin du Moyen Âge. Thèse de l'École nationale des chartes. Paris : 2008.

18. - JEHANNO, Christine. «Les comptes de l'Hôtel-Dieu de Paris au Moyen Âge. Documents pour servir à l'histoire économique et sociale ». Études et documents. Comité pour l'histoire économique et financière de la France, IX, 1997, p. 503-527.

19. - FIANU, Kouky, FORTIER, Anne, CLAVAUD, Florence (dir.). L'Année 1437 dans la pratique de Pierre Christofle, notaire du Châtelet d'Orléans. École nationale des chartes, Paris, 2015. Voir le site : http://elec.enc.sorbonne.fr/christofle/ [consulté le 18/07/2019].

20. - « Traversain » a ici le sens de « petit tonneau ».

21. - Voir Annexe 1: Contrat de mariage entre Jehan Gaultier et Jehanne Toubert, année 1450, étude Filleul. AD Loiret, 3E10158, fol. 5.

22. - Voir Annexe 2 : Contrat de mariage entre Jehan Blanchart et Marion Piquart, 4 mai 1449, étude Christofle. AD Loiret, 3E10150, fol. 5v.

23. - AD Loiret, 3E10150, fol. $96 \mathrm{v}^{\circ}, 6$ mai 1437, Étude Christofle, édité par Kouky Fianu. L'année 1437 dans la pratique de Pierre Christofle, notaire du Châtelet d'Orléans. Voir le site: http:// elec.enc.sorbonne.fr/christofle/ [consulté le 18/07/2019].

24. - Voir Annexe 3 : Reconnaissance de dette et gage entre Colete, veuve Miracle et maîtres Mynart et Libart, 25 juin 1437, étude Giraut. AD Loiret, 3E10138, fol. 192.

25. - Voir Annexe 4: prêt de mobilier entre David Jubin, boulanger et Jaquet Le Maistre, tavernier, 29 décembre 1481. AD Yvelines, 3 E 48-38, fol. 315.

26. - Voir Annexe 5 : prêt de mobilier entre David Jubin, boulanger et Jaquet Le Maistre, hôtelier, 7 février 1482 n. st. AD Yvelines, 3 E 48-38, fol. 331.

27. - DUTOUR, Thierry. Sous l'empire du bien «Bonnes gens" et pacte social (XIII -XVe siècle). Paris : Classiques Garnier, 2015, p. 139-142. 
28. - KARPIK, Lucien. "L'économie de la qualité ». Revue française de sociologie, 1989, vol. 30, p. 206-207.

\section{RÉSUMÉS}

Cet article étudie la place symbolique prépondérante du lit dans la société médiévale des XIV et $\mathrm{XV}^{\mathrm{e}}$ siècles, en partant de sources textuelles éditées et inédites, en particulier les inventaires après décès et les testaments. En effet, nombre d'actes notariés dépouillés ont pu faire état de pratiques sociales jusqu'ici obscures ou ignorées de l'histoire matérielle. En examinant des actes d'une grande variété, l'objectif de cet article n'est pas de fournir des réponses définitives mais bien de proposer de nouveaux angles d'approche sur l'histoire du mobilier en France à la fin du Moyen Âge et un éclairage particulier sur le rôle central du lit dans les relations de sociabilité de cette époque.

This paper focuses on the symbolic role of the bed in the medieval XIVth and XVth centuries society, on the basis of written sources, particularly after death inventories and wills. Indeed, numerous deeds have shown social practices so far uncleared or ignored in material history. In treating a wide variety of deeds, this paper's objective isn't to bring definite answers but to propose new approaches about furniture history in France in the late medieval era, and a particular perspective on the bed's central role in sociability relationships of this period.

\section{INDEX}

Keywords : furniture, bed, material culture, social organization, text editing, after death inventory, testament, deeds, Departmental archives, donation, everyday life, contracts, sociability, Middle Ages, medieval housing, medieval house

Mots-clés : mobilier, lit, culture matérielle, organisation sociale, édition de textes, inventaire après décès, testament, actes notariés, archives départementales, donation, vie quotidienne, contrats, sociabilité, Moyen Âge, habitat médiéval, maison médiévale

\section{AUTEUR}

\section{DONATIEN GUÉGAN}

Architecte HMONP, Sorbonne Université, master d'histoire « Mondes médiévaux » donatien.guegan@yahoo.fr 